\title{
Multiple Conformations of Compact Dinucleosomes: Analysis by Electron Microscopy
}

Maria Valieva $^{1}$, Oleg Chertkov ${ }^{1}$, Maria Karlova ${ }^{1}$, Mikhail P. Kirpichnikov ${ }^{1}$, Alexey V. Feofanov ${ }^{1,2}$, Olga S. Sokolova ${ }^{1}$ and Vasily Studitsky ${ }^{1,3}$

1. Biology Faculty, Lomonosov, Moscow State University, Moscow, 119992, Russia

2. Shemyakin-Ovchinnikov Institute of Bioorganic Chemistry of Russian Academy of Sciences, 117997 Moscow, Russia

3. Fox Chase Cancer Center, Philadelphia, PA, 19111-2497, USA

The packaging of genomic DNA into chromatin has a significant impact on numerous key processes in the cell, including replication, repair and transcription $[1,2]$. Each nucleosome consists of 147 pairs of DNA nucleotides wrapped around and octamer of core histones [3]. In the cell nuclei, nucleosomes can move along DNA spontaneously and with help of ATP-dependent chromatin remodeling factors [4-6]. As a result, closely positioned and overlapping dinucleosomes and nucleosome-free DNA regions can be formed, most often at regulatory regions of the genome [7]. It has been proposed that closely positioned and overlapping dinucleosomes could be unstable and play a regulatory role in vivo. Structures of the dinucleosomes have been modeled and studied using adjacent nucleosome positioning sequences having high-affinity to core histones [7, 8, 9]. However, the structure and dynamics of compact dinucleosomes formed on DNA sequences having a lower, more physiological affinity to core histones have not been studied.

In our study, the structure and dynamics of the positioned nucleosome collided with the second nucleosome formed on a natural DNA sequence were analyzed. Nucleosomes were reconstituted by salt dialysis in the presence of -H1 chromatin from chicken erythrocytes, and then purified from a nondenaturing gel (Fig. 1A). To investigate the structural heterogeneity of the dinucleosomes, purified from a single band on the non-denaturing gel, gel-purified dinucleosomes were analyzed using electron microscopy after negative staining (Fig. 1B). Images were taken using JEM2100 microscope (JEOL) (magnification $\mathrm{x} 40,000)$. The 1300 dinucleosome particles were semi-automatically collected using BOXER [10] and classified into 100 2D classes using EMAN [10]. Then the distances between centers of the two visible particles in all single complexes were measured using ImageJ program (Fig.1C).

The observed differences in distances between centres of the particles revealed that the compact dinucleosomes have variable structures with the range of distances between the particles of 10-22 nm. Interestingly, the distances between the particles in the most favourable conformations are changed stepwise, with 3-4 nm increment (approximately one turn of the double helix, $3.6 \mathrm{~nm}$ ), corresponding to distances of $\sim 11,15$ or $18 \mathrm{~nm}$ (Fig. 1C). The data suggest that DNA in the dinucleosome is uncoiled from the histone octamer stepwise, one turn of the DNA helix at the time. In the complexes characterized by a larger distance between the particles (presumably containing DNA that is more uncoiled from one of the octamers) one nucleosome projection was smaller than the other (Fig 1B, complexes 2,3). The data suggest that histone content in one of the two nucleosomes was changed, possibly due to transient loss of an $\mathrm{H} 2 \mathrm{~A} / \mathrm{H} 2 \mathrm{~B}$ dimer from nucleosomes containing uncoiled DNA.

In conclusion, our results suggest that compact dinucleosomes are dynamic structures containing nucleosomes having variable structure and stability. In each constituent nucleosome DNA can be 
reversibly uncoiled from the histone octamer by 10 or $20 \mathrm{bp}$, resulting in formation of less stable dinucleosomes.

References:

[1] Venkatesh, S. and Workman, J. L. Nat Rev Mol Cell Biol 16 (2015), p. 178.

[2] Talbert, P. B. and Henikoff, S. Nat Rev Mol Cell Biol 18 (2017), p. 115.

[3] Davey, C. A et al. J Mol Biol 319 (2002), p. 1097.

[4] Bouazoune et al. Nucleic Acids Res. 37(16) (2009), p. 5279.

[5] Clapier C.R. and Cairns B.R. Annu Rev Biochem. 78 (2009), p. 273.

[6] Mueller-Planitz F., Klinker H., Becker P.B. Nat Struct Mol Biol. 20(9) (2013), p. 1026.

[7] Kato, D. et al. Science 356 (2017), 205.

[8] Ulyanova N.P., Schnitzler G.R. Mol Cell Biol. 25(24) (2005), p. 11156.

[9] Engeholm et al, Nat Struct Mol Biol. 16(2) (2009), p. 151.

[10] Tang et al. J Struct Biol. 157 (2007), p. 38.

[11] The authors acknowledge funding from the Russian Science Foundation (Grant \#14-24-00031).
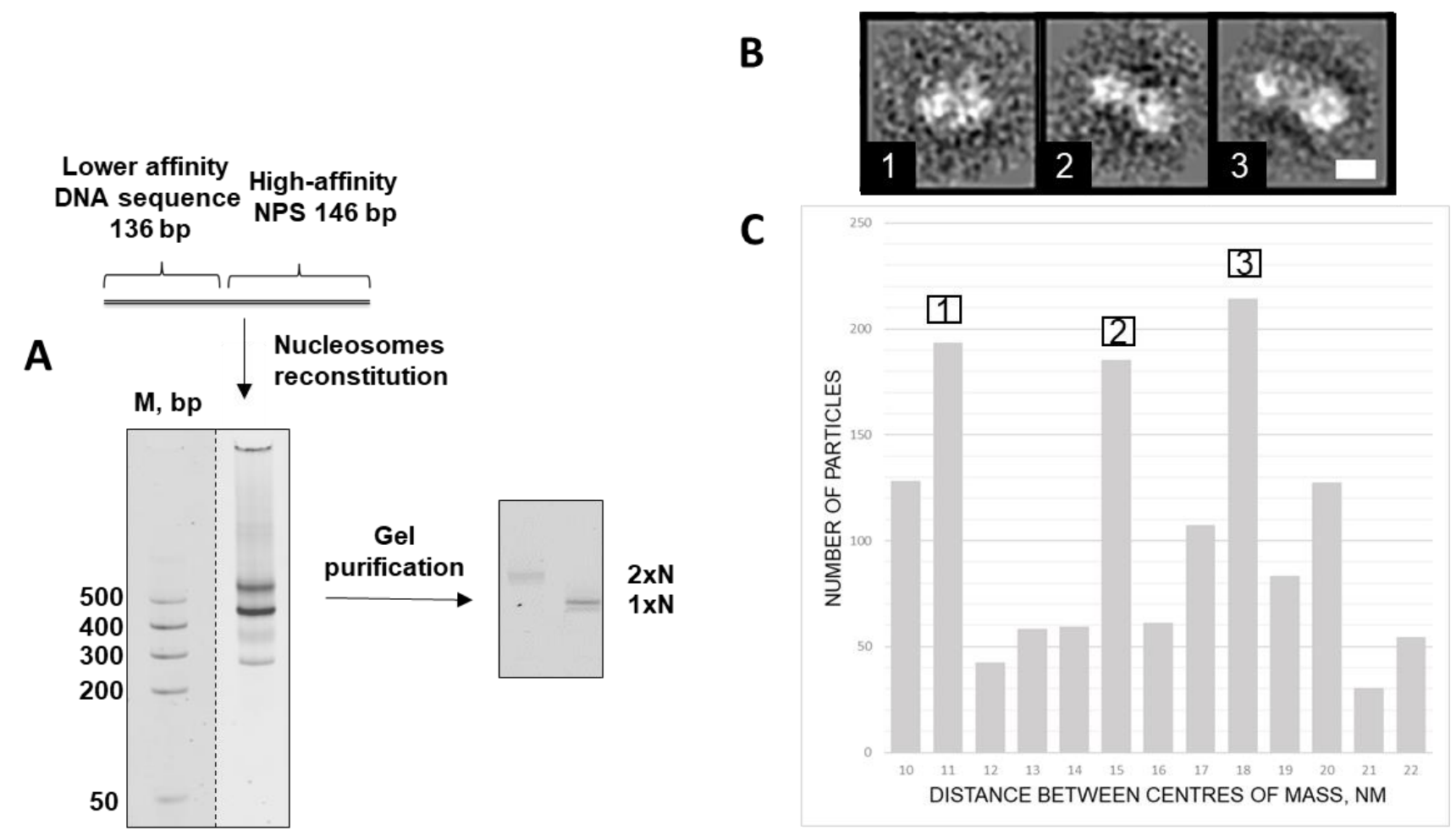

Figure 1. A dynamic nature of compact dinucleosomes. (A) Sample preparation. Mono- $(1 \mathrm{xN})$ and dinucleosomes $(2 \mathrm{xN})$ were assembled on the DNA template, containing the 603 nucleosome positioning sequence (NPS) having high affinity to core histones and a 136-bp natural DNA sequence. (B) Representative 2D class averages of dinucleosomes with different distances between centers of masses. Scale bar $10 \mathrm{~nm}$. (C) Distribution of distances between centers of masses in all studied dinucleosomes. Numbers correspond to the class averages in (B). 